\title{
TM-mode AlGaInAs/InP Amplifying Waveguide Optical Isolator with 12.7dB Isolation
}

\author{
W. Van Parys, D. Van Thourhout and R. Baets \\ Department of Information Technology (INTEC), Ghent University-IMEC, Sint-Pietersnieuwstraat 41, 9000, Gent, Belgium \\ Wouter.VanParys@intec.UGent.be \\ B. Dagens, J. Decobert, O. Le Gouezigou and D. Make \\ Alcatel Thales III-V Lab, Route Départementale 12891767 Palaiseau, France \\ R. Vanheertum and L. Lagae \\ Interuniversitair Micro Electronica Centrum IMEC vzw, Kapeldreef 75, B-3001 Leuven, Belgium
}

\begin{abstract}
We present the development of a monolithically integratable optical isolator. The device is a semiconductor optical amplifier with magnetized ferromagnetic metal contact. $12.7 \mathrm{~dB}$ isolation and optical transparency are demonstrated. The spectral dependence is studied.

(C)2005 Optical Society of America

OCIS codes: (230.3240) Isolators; (250.5980) Semiconductor optical amplifiers; (230.3810) Magneto-optical devices
\end{abstract}

\section{Introduction}

An optical isolator is indispensable for the protection of a laser source from the optical feedback in a telecom link. A planar, waveguide version of this component is highly desirable as this would greatly reduce the cost and size of a laser diode package. Traditionally, research focuses on ferrimagnetic garnet material to realize non-reciprocity [1,2]. Stand-alone isolators with good performance have been demonstrated but the integration with III-V semiconductor hosts remains an issue that is difficult to solve.

We are studying a TM-mode isolator configuration which holds the promise of monolithic integration with III-V active devices [3,4]. Recently, a variant for TE-polarization has been proposed and demonstrated [5]. Here, we report on the design, fabrication and characterization of an optical isolator with largely improved performance. Furthermore, the spectral dependence of the isolator performance is studied.

\section{Theoretical Concept}
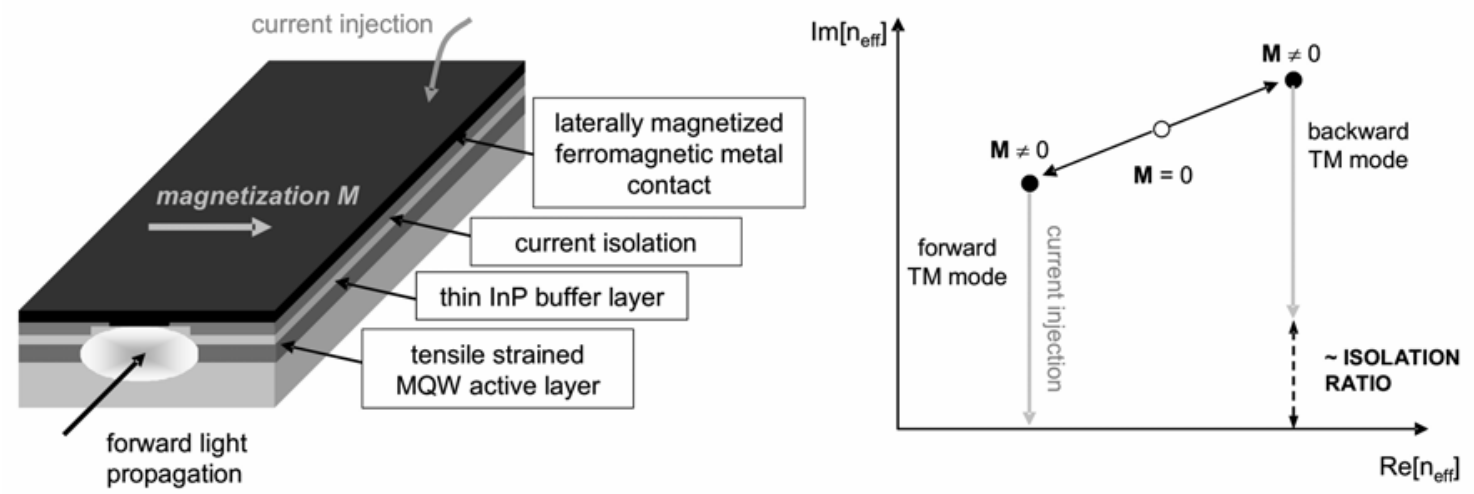

Fig. 1. Schematic lay-out and operation principle of the TM-mode optical waveguide isolator.

The amplifying waveguide isolator is a semiconductor optical amplifier (SOA) topped with a laterally magnetized (magnetization $\mathbf{M} \neq 0$ ) ferromagnetic metal film, which is separated from the amplifying core by only a very narrow cladding layer. The magneto-optic Kerr effect causes a non-recipocal complex shift of the effective index of TMpolarized guided modes. Electrical pumping of the SOA with the ferromagnetic metal as the electrical contact compensates the loss in the forward propagation direction. The result is a device, which, being transparent in one propagation direction while providing net loss in the other direction, is isolating. Figure 1 illustrates the lay-out and the operation principle of the isolator. As this device basically has the same structure as the laser it is to be integrated with, monolithic integration is straightforward. Furthermore, as the ferromagnetic film can easily be 


\section{ITuG3.pdf}

sputter-deposited on III-V semiconductor layers, the optical waveguide isolator can be fabricated using standard InP-SOA processing techniques.

\section{Design and fabrication}

The active material used to compensate the forward modal loss is an optimized AlGaInAs/InP tensile strained multiquantum well structure. Built-in tensile strain realizes TM-selective material gain, while TE gain is suppressed. The number of quantum wells (9QWs) and the amount of tensile strain (-1.16\%) in the wells and compressive strain in the strain compensating barriers $(+0.64 \%)$ have been optimized for optimal gain properties [6]. The ferromagnetic metal film fulfills two functions - it is the source of the non-reciprocal effect and it provides the electrical contact for the underlying SOA. The complex optical and magneto-optic parameters of a number of $\mathrm{Co}_{\mathrm{x}} \mathrm{Fe}_{1-\mathrm{x}}$ alloys $(\mathrm{x}=90$, 50,0 ) have been experimentally extracted at the operation wavelength of $1300 \mathrm{~nm}$. It was found that the equiatomic $\mathrm{Co}_{50} \mathrm{Fe}_{50}$ alloy provides the best combination between high magneto-optic strength and low optical absorption, hence it is the preferred material [7]. A $\mathrm{p}^{++}$-doped semiconductor contact layer has been designed that realizes an ohmic electrical contact without significantly increasing the modal absorption.

Apart from these main building blocks, the isolator performance is determined by the dimensions of three cladding layers: the upper and lower separate confinement heterostructure $(\mathrm{SCH})$ layers surrounding the quantum well material and the InP cladding between the waveguide core and the contact structure. Contrary to previous approaches where the isolation ratio (in $\mathrm{dB} / \mathrm{cm}$ ) was maximized [4], the current design strategy focuses on the practical specifications of the isolator. In other words, the figure of merit to be minimized is the total current needed for forward transparency, corresponding to a fixed value of the optical isolation. 1D simulations have been done with an optical mode solver [8] extended with an algorithm for magneto-optic waveguide calculation [9]. The experimentally determined gain-current density relation of the amplifying material and the extracted parameters of $\mathrm{Co}_{50} \mathrm{Fe}_{50}$ served as input for these simulations. A steepest descend algorithm rapidly resulted in the best thickness combination. Theoretically, $62 \mathrm{~mA}$ suffices to achieve transparency on a $2 \mathrm{~mm}$ long isolator providing $10 \mathrm{~dB}$ isolation.

The optimized active material has been grown by metal organic vapor phase epitaxy (MOVPE) on an InP substrate. It was topped with a $400 \mathrm{~nm}$ InP cladding layer and the $\mathrm{p}^{++}$-doped contact structure. A 50nm $\mathrm{Co}_{50} \mathrm{Fe}_{50}$ film was sputter-deposited and covered with a protective Ti/Au bilayer. The metal layers were patterned into $2 \mu \mathrm{m}$ wide stripes through standard lift-off and subsequently used as an etch mask for the definition of the ridge waveguides, with RIE etching. With this procedure full covering of the ridges with metal has been achieved.

\section{Characterization}

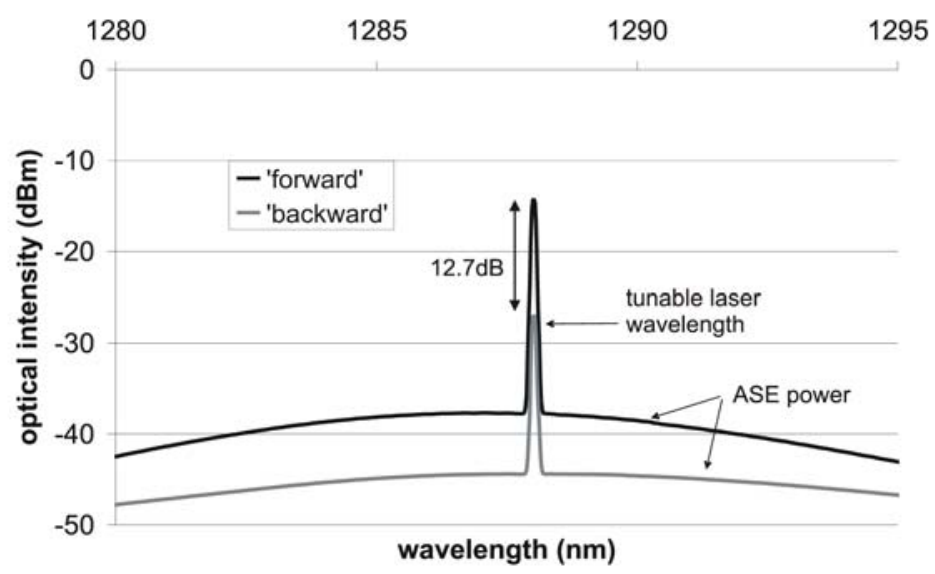

Fig. 2. Spectrum of the output signal at saturation magnetization in both lateral directions, showing $12.7 \mathrm{~dB}$ optical isolation; measured with 160mA bias current (ASE = amplified spontaneous emission).

The characterization of this non-reciprocal device is done by evaluation of TM-polarized transmitted light at saturation magnetization in both lateral directions - changing the magnetic field direction is equivalent to switching between forward and backward propagation direction. A measurement example is presented in figure 2. The input light comes from an external cavity tunable laser $(-10 \mathrm{dBm}$ power) and the signal is evaluated on a spectrum analyzer (resolution bandwidth $0.1 \mathrm{~nm}$ ). The cavity length equals $2 \mathrm{~mm}$ and broadband AR-coatings have been deposited on the chip facets. The CW bias current equals 160mA. The difference in output power between the 'forward' and 'backward' signal - the optical isolation - equals $12.7 \mathrm{~dB}$. The total insertion loss in 'forward' propagation direction 


\section{ITuG3.pdf}

equals 4.6dB. The fiber-to-chip coupling losses are measured to be 4 to $5 \mathrm{~dB}$ per facet. It can therefore be concluded that at $160 \mathrm{~mA}$ current injection, on-chip amplification in the forward propagation direction has been achieved. Comparing the demonstrated performance $-12.7 \mathrm{~dB}$ isolation combined with forward transparency below $160 \mathrm{~mA}$ with what the 1D simulations predicted - 10dB isolation and a transparency bias current of $62 \mathrm{~mA}$ - shows that both the experimental isolation and modal loss are higher. This discrepancy between theory and experiment is believed to result mainly from a reduction of the thickness of the InP cladding layer, which increases the modal overlap with the ferromagnetic metal, resulting in higher value for both the non-reciprocal effect and the modal absorption. This issue needs further investigation. The use of $1 \mathrm{D}$ simulations to calculate the theoretical isolator performance adds an extra difference between simulation and experiment.

Repeating this measurement of isolation and insertion loss for different tunable laser wavelengths results in figure 3, illustrating the spectral dependence of the isolator. The values for the insertion loss are for 'forward' propagating light and include the fiber-to-chip coupling losses. The optical isolation changes 3.6dB within a wavelength range of $16 \mathrm{~nm}$. The wavelength dependence of the insertion loss, which obviously is directly correlated to the gain spectrum of the quantum well material, is much larger and is the dominating factor in the spectral dependence of the isolator performance.

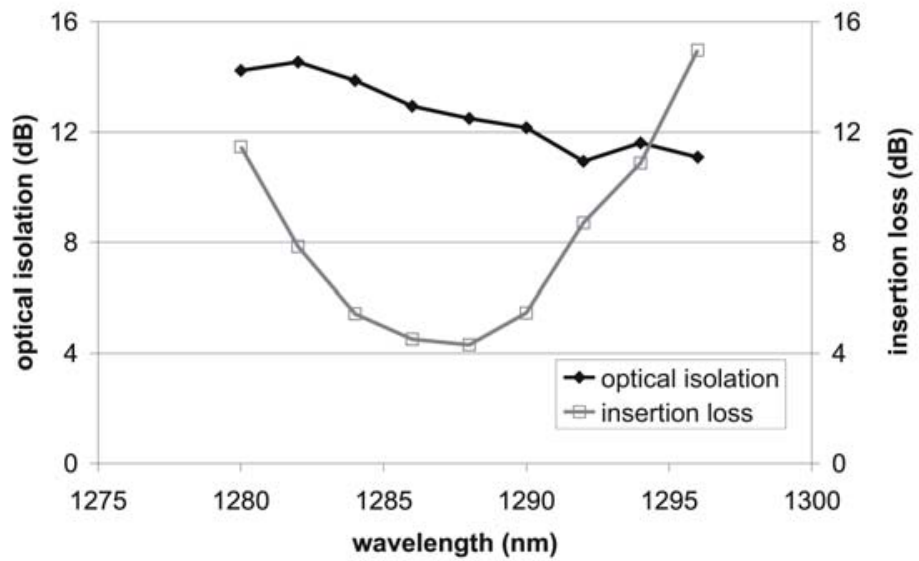

Fig. 3. Spectral dependence of the optical isolation and insertion loss of the waveguide isolator.

\section{Conclusion}

In conclusion, we have demonstrated a monolithically integratable optical isolator with $12.7 \mathrm{~dB}$ optical isolation. Forward transparency has been achieved for a bias current below 160mA. It was shown that the wavelength dependence of the isolator is mainly determined by the gain spectrum of the amplifying material.

\section{References}

[1] H. Dötsch, N. Bahlmann, O. Zhuromskyy, M. Hammer, L. Wilkens, R. Gerhardt, and P. Hertel, “Applications of Magneto-Optical Waveguides in Integrated Optics: Review”, JOSA B 22, 240-253 (2005).

[2] H. Yokoi, T. Mizumoto, N. Shinjo, N. Futakuchi, and Y. Nakano, "Demonstration of an optical isolator with a semiconductor guiding layer that was obtained by use of a nonreciprocal phase shift, Appl. Opt. 39, 6158-6164 (2000).

[3] M. Takenaka, and Y. Nakano, "Proposal of a Novel Semiconductor Optical Waveguide Isolator", in Proc. 11 ${ }^{\text {th }}$ Int. Conf. on Indium Phosphide and Related Materials (Institute of Electrical and Electronics Engineers, New York, 1999), pp.289-292.

[4] M. Vanwolleghem, W. Van Parys, D. Van Thourhout, R. Baets, F. Lelarge, O. Gauthier-Lafaye, B. Thedrez, R. Wirix-Speetjens, and L. Lagae, "Experimental demonstration of nonreciprocal amplified spontaneous emission in a CoFe clad semiconductor optical amplifier for use as an integrated optical isolator”, APL 85, 3980-3982 (2004).

[5] H. Shimizu, and Y. Nakano, “14.7dB/mm TE Mode Nonreciprocal Propagation in an InGaAsP/InP Active Waveguide Optical Isolator”, in Optical Fiber Communication Conference (The Optical Society of America, Washington, DC, 2005), paper PDP18.

[6] J. Decobert, N. Lagay, C. Cuisin, B. Dagens, B. Thedrez, F. Laruelle, "MOVPE Growth of AlGaInAs-InP Highly Tensile Strained MQWs for 1.3 $\mu \mathrm{m}$ Low-Threshold Lasers”, J. Crystal Growth 272, 542-548 (2004).

[7] M. Vanwolleghem, W. Van Parys, P. Gogol, P. Beauvillain, and R. Baets, "Design and optimization of a monolithically integrated InP-based optical waveguide isolator”, submitted to JOSA B.

[8] P. Bienstman and R. Baets, "Optical modelling of photonic crystals and VCSELs using eigenmode expansion and perfectly matched layers", Opt. and Quant. Elect. 33, 327-341 (2001).

[9] K. Postava, M. Vanwolleghem, D. Van Thourhout, R. Baets, S. Visnovský, P. Beauvillain, J. Pistora, “Modeling of a novel InP-based monolithically integrated magneto-optical waveguide isolator”, JOSA B 22, 261-273 (2005). 